\title{
Point Cloud Segmentation to Approximately Convex Surfaces for Fruit Recognition
}

\author{
Robert Cupec, Damir Filko, Ivan Vidović, Emmanuel Karlo Nyarko, Željko Hocenski \\ Faculty of Electrical Engineering \\ J. J. Strossmayer University of Osijek \\ Osijek, Croatia \\ robert.cupec@etfos.hr
}

\begin{abstract}
A fruit recognition approach based on segmenting the point cloud acquired by a $3 D$ camera into approximately convex surfaces is considered. A segmentation approach which transforms a depth image into a triangular mesh and then segments this mesh into approximately convex segments is applied to depth images of fruits on trees. An analysis of the results obtained by this approach is performed with the intention to determine how successful the studied method is in detecting fruit as separate objects in a point cloud. The reported analysis gives a valuable insight into the potential applicability of the tested methodology in the preprocessing stage of a fruit recognition system as well as its drawbacks.
\end{abstract}

Keywords-fruit recognition;3D camera; convex sets; segmentation

\section{INTRODUCTION}

The very purpose of robotics and automation is to free the humans from heavy, tedious and repetitive work. Although many production tasks which had been done by humans in the past are today performed by machines, there are many operations in industrial and agricultural production process which still require human involvement due to the lack of suitable technical solutions. Tasks like textile handling, fruit picking and many assembling tasks, although rather simple for a human, represent a real challenge for a machine. Solving such tasks requires highly advanced environment perception capable of interpreting unstructured scenes and objects of varying shapes.

In this paper, robotized fruit picking problem is considered. In order to solve this task, a robot must be capable of recognizing fruit on trees, usually occluded by leaves and branches and under different lighting conditions. Unlike office or household objects which are commonly used as case studies in object recognition research [28] - [30], the shape, size and color of fruit varies among samples of the same sort, which makes their recognition more difficult. A robust and efficient fruit recognition method should exploit both shape and color properties of fruit.

The fruit recognition approach studied in this paper relies on the fact that the shape of many fruit sorts can be approximated by convex shapes. The proposed approach consists of segmenting the point cloud acquired by a 3D camera into approximately convex surfaces and applying an appropriate classifier based on color and shape in order to distinguish between the fruit and other objects such as leaves and branches. The focus of this paper is on the segmentation stage, while the selection of an appropriate classification method is not covered. The results of the point cloud segmentation method proposed in [1] are analyzed in order to assess its applicability in a fruit recognition scheme. In an ideal case, the applied method should provide such point cloud segmentation where each fruit is represented by a single approximately convex surface. In reality, however, due to a limited sensor accuracy and measurement noise, the points representing a fruit can be merged with the points of adjacent leaves or branches into an approximately convex surface which exceeds the boundaries of the considered fruit. Furthermore, due to occlusion and gaps in the point cloud caused by light absorption effects and specular reflection, the set of points representing a single fruit can be split into two or more mutually disconnected subsets resulting in oversegmentation. In order to evaluate the applicability of the investigated segmentation approach, an experimental analysis is performed whose goal is to determine the percentage of successful segmentation results, i.e. the cases where a fruit is represented by a single segment, as well as the percentage of false segmentation results, where a single fruit is represented by two or more segments or included in a segment which extends to adjacent objects.

The paper is structured as follows. The overview of the research in the field of fruit recognition is given in Section II. The segmentation method which is in focus of this paper is described in Section III. In Section IV, an experimental analysis of the applicability of the considered segmentation method for usage in a fruit recognition system is presented. The discussion of the obtained results and some possible directions for future research are given in Section V.

\section{RELATED RESEARCH}

Fruit detection, recognition or localization on trees has been subject of many scientific papers. Fruit recognition systems are used for different applications, like automatic harvesting, yield estimation, phenotyping, breeding, etc. There are many different approaches to solve the mentioned problems and some of them are listed below. 
One of the first papers in this field is [2] and it proposes a method for fruit size and location detection. The method is based on image color information and thus retraining is necessary every time when fruit color changes significantly. The detection accuracy under favorable conditions can reach up to $75 \%$. Another color based approach for automatic recognition of Fuji apple based on red color difference and finding the maximum between-class variance in histogram is given in [3]. Unlike [2], the algorithm is not sensitive to lightening conditions, but because it relies on red color difference it is limited to Fuji apples or similar color fruit recognition. The obtained successful recogniton was over $88 \%$, but average error rate reached up to $18 \%$. Detection of citrus fruits using fusion of color map and luminance map is proposed in [4]. The recognition accuracy under different lightening conditions was up to $86,81 \%$ with a false detection rate of 2,25\%. Forward feature selection algorithm (FFSA) for color feature selection is used in [5]. Different classifiers are applied on selected features to seperate blueberry fruit from the background and to classify the detected fruits according to matureness. A mature citrus recognition method based on $\mathrm{YCbCr}$ color model is proposed in [6]. Using an improved fuzzy C-means algorithm, morphological operations and optimized Circular Hough transform (CHT), fruits center and radius are detected. Algorithm for citrus fruit and obstacles recognition based on multi-class support vector machine applied on color features is proposed in [7]. The recognition rate was $92,4 \%$ and branches with diameter larger than 5 pixels were also detected.

Instead of color features, authors in [8] used fruit shape prosperities for wine grape berries recognition in controled environment. After the image preprocessing, CHT is applied for berries detection. Wine grape berries sizes estimation is discussed in [9]. First berries are detected using CHT, then histogram of oriented gradients $(\mathrm{HoG})$ and gist features are extracted followed by application of conditional random field for classifying circles as berry and non-berry. Experiments showed on average $1 \mathrm{~mm}$ difference of estimated size compared to manual measurement. The authors also mentioned possible usage of depth image to precisely determine berries size. Immature citrus recognition and counting based on shape analysis and texture classification is proposed in [10]. Shape analysis based on CHT is used to detect circles while texture classification is used for false positives removal. Algorithms used for texture analysis are: support vector machine (SVM), Canny edge detection combined with a graph-based connected component algorithm, Hough line detection and scale invariant feature points (SIFT) algorithm. The method is tested on images captured in natural outdoor conditions and recognition and counting rate was $80,4 \%$.

In [11] a method for recognizing and counting peppers in cluttered greenhouse is presented. The proposed method is a two-step approach based on detecting peppers from multiple views and applying a statistical method for combining the detection results. Experiments are performed on 28000 images and $74,2 \%$ accuracy of detection is achieved. A new method for grapevine berries size and weight determination and it's extension to detection of other fruits is proposed in [12]. The first step of the proposed method is peduncle detection and it is based on a novel signature of the contour. The reported experiments show that the grapevine berry weight and size can be correctly estimated with correlation coeficient $R^{2}>0,96$ and $\mathrm{R}^{2}>0,97$ respectively. For other fruits, estimation of the size is also accurate with $\mathrm{R}^{2}>0,93$. A vision system for automatic apple harvesting is proposed in [13]. The method consists of four steps: preprocessing by vector median filter, image segmentation by seeded region growing method using color features, color and shape feature extraction and fruit detection using SVM. In the reported experiments $89 \%$ of apples were successfully detected. The idea of applying convexity analysis in fruit detection is used in [14], where intensity model is created from 2D image and a convexity test is applied to that model in order to detect apples on trees. The proposed method detected $94 \%$ of visible apples, while $14 \%$ of the identified objects were false positives.

Apple fruit counting and diameter estimation using thermal image is proposed in [15]. The captured image is transformed using normal difference index (NDI) and after that morphological operations, feature extraction, particle selection and classifier evaluation are applied. The correlation coefficient with manual measuring was 0,88 and 0,70 for apple counting and diameter estimation respectively. A drawback of the method is recognizing the fruits growing deep in the treecrown and the authors sugest usage of some shape detection algorithm for the mentioned problem. Fusion of vision and thermal images for oranges detection is proposed in [16]. After image registration, Laplacian pyramid transform (LPT) and fuzzy logic are used for image fusion. It is shown that fuzzy logic performs better and that image fusion improves fruit detection when visible image was over-exposed or the fruit was warmer.

In addition to the approaches mentioned so far which are based on 2D images, many methods which use stereo vision and 3D sensors are also proposed. In [17] stereo vision is used for apple and pear detection and automatic harvesting. Detection is based on a color intensity threshold and Linear Color Models (LCM). The method is tested only in controlled laboratory conditions. To overcome problems with uneven illumination, partly occluded surfaces and similar background features, the authors in [18] used a combination of the object's color, texture and 3D shape properties. Recognition of clustered tomatoes using binocular stereovision is proposed in [19]. The method is based on depth map filtering, Otsu thresholding and edge curvature analysis. The recognition accuracy of the clustered tomatoes was $87,9 \%$ when the leaf or branch occlusion rate was less than $25 \%$. Localization of oranges for automatic harvesting is proposed in [20]. Harvesting is based on matching of oranges detected in stereo images acquired from two robot arms and on applying double traveling salesman problem (DTSP) for arm path computation.

A laser based computer vision system for automatic orange harvesting is proposed in [21]. Both range and reflectance information is used to generate four characteristic primitives: contour pixels, crown pixels, convex regions and reflectivitybased regions. The generated primitives are used for spherical object recognition and localization. The main drawback of the proposed method is the image acquisition time which is 25 seconds for a $160 \times 160$ image. Another laser based approach is 
proposed in [22]. The applied sensor is equipped with red and infrared laser diodes and both beams scan an object simultaneously. Cherries are detected using the difference in the spectral reflection characteristics between the laser beams.

Good surveys on computer vision methods and algorithms used in automation of fruit production process can be found in [23] - [26].

Analogously to the research presented in [14], the approach proposed in this paper relies on the convexity property of the fruit. However, instead of analyzing intensity profiles between two edge points in order to detect convex surfaces in 2D image, we use a 3D camera and detect convex surfaces in the obtained point clouds. We believe that by application of a 3D camera a more robust performance can be achieved, because the detection of convex surfaces in point clouds acquired by such cameras is less dependent on illumination conditions.

In comparison to the work presented in [21], where laser sensors are applied to detect geometric structures in the scene, we investigate the application of a low-cost 3D camera which acquires point clouds with a frame rate of $30 \mathrm{fps}$. Thereby, a much faster detection of convex structures is achieved. On the other hand, the sensor used in the experiments presented in this paper provides less accurate measurements then the equipment applied in [21] and we want to determine how useful the measurements obtained by such sensor are in the context of fruit recognition.

\section{Segmentation of 3D Point Clouds INTO APPROXIMATELY CONVEX SURFACES}

A point cloud obtained by a $3 \mathrm{D}$ camera such as Kinect or PrimeSense sensor represents a set of 3D points lying on the scene surfaces visible from a particular viewpoint. Each of these points is the closest point to the camera in a particular direction, i.e. there are no solid non-transparent objects between a point in a considered point cloud and the camera. Hence, each point in the point cloud projects onto the different point on the camera image plane. Such a point cloud can therefore be regarded as a depth image, i.e. an image where each pixel is assigned its distance from the camera. An example of an RGB image and the corresponding depth image is shown in Fig. 1. (a) and (b) respectively. The depth image is presented in grayscale, where the pixel intensity is proportional to the depth except in the case of black color which represents the pixels which are not assigned a depth value.

The efficient point cloud segmentation into approximately convex surfaces proposed in [1] requires a preprocessing step in which a triangular mesh is built from the point cloud. A triangular mesh is a representation of a point cloud by a set of triangles such that for each point in the point cloud there is a triangle whose distance to this point is less than a predefined threshold value. By representing a point cloud with a triangular mesh a reduction of data is achieved since a triangle defined by three points usually represents a set of many points of the point cloud. This data reduction results in computation time saving. It is especially efficient for scenes dominated by low curvature surfaces, e.g. indoor scenes with large planar surfaces. To generate a triangular mesh from a point cloud we use the algorithm based on recursive triangulation refinement resulting

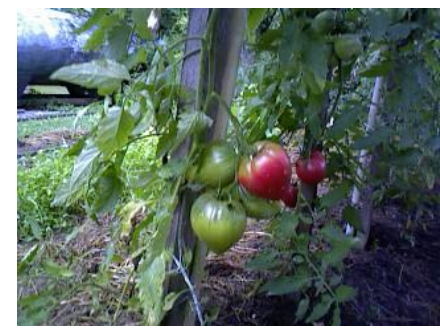

(a)

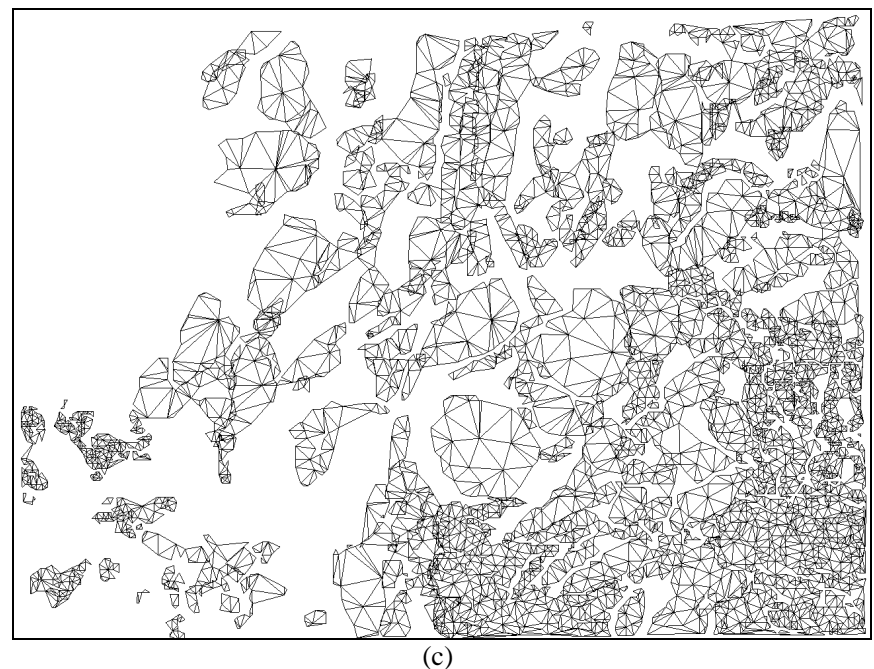

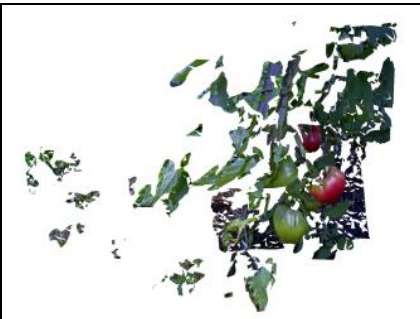

(d)

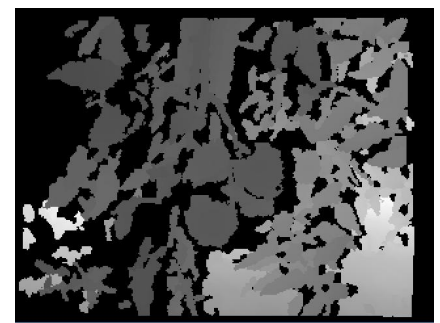

(b)

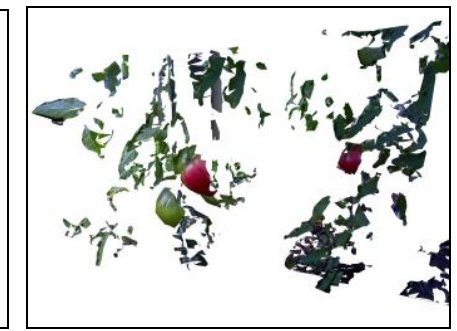

(e)
Fig. 1. (a) Sample RGB image; (b) corresponding depth image; (c) triangular mesh; (d), (e) triangular mesh with the RGB image projected onto it presented in two different views.

in a Delaunay triangulation proposed in [27]. After the triangulation is completed, each triangle for which the angle between its normal and the optical ray corresponding to one of its vertices is greater than $73^{\circ}$ is rejected from the mesh because such triangles mostly represent depth discontinuities rather than an object surface. Furthermore, all triangles with less than $50 \%$ supporting image points not assigned depth are also rejected from the mesh, because they mostly represent artefacts bridging gaps in the depth map rather than true surfaces present in the scene. The triangular mesh created from the depth image shown in Fig. 1. (b) is presented in Fig. 1. (c). The obtained 3D model of the scene is visualized in Fig. 1. (d) and (e) by projecting the RGB image shown in Fig. 1. (b) onto the triangular mesh shown in Fig. 1. (c).

The triangular mesh is then segmented into approximately convex surfaces. A method which builds a hierarchical convex approximation of 3D shapes given a tetrahedral mesh is proposed in [31]. Since a software implementation of that method is not publically available, it is hard to assess its 
computational efficiency. Nevertheless, from the data provided in [31], we estimated that the method proposed in [1] is faster, hence we used this method.

The procedure for segmentation of a triangular mesh applied in [1] is described in the following. First, the largest triangle is selected. This triangle represents the initial singletriangle set which is expanded by an iterative region growing procedure. In each iteration, a triangle adjacent to one of the triangles in the expanding set is added to this set, while preserving the property of the set that the distance of every triangle in the set from the convex hull of the set is below a predefined threshold. The region growing procedure stops, when no additional triangle can be appended to the considered set without losing the aforementioned approximate convexity property. After one segment is completed, the algorithm continues applying the same procedure to the remaining triangles which are not assigned to any segment. The described segmentation approach is presented as Algorithm 1 given in the following.

Algorithm 1: Point Cloud Segmentation into Approximately Convex Surfaces

\section{Input: Depth image, $\varepsilon$}

Output: Set of segments $\Sigma$

1 Create triangular mesh $A$ from the input depth image using the recursive triangulation refinement method proposed in [27].

$2 \quad A^{\prime} \leftarrow A$.

3 Repeat

4

5

6

\section{Return $\Sigma$.}

A detailed explanation of how to compute the convex hull in line 16 and how the distance between a segment and its convex hull in line 17 is defined is given in [1]. The output of the presented algorithm is the set $\Sigma$ of segments representing approximately convex surfaces. How close a surface must be to its convex hull in order to be considered approximately convex is specified by a user defined parameter $\varepsilon$. An example of segmentation to approximately convex surfaces is shown in Fig. 2, where segments obtained by applying the described method to the triangular mesh shown in Fig. 1. (c) are displayed.

\section{EXPERIMENT}

In this section an analysis of the results obtained by applying the segmentation algorithm proposed in [1] to RGB-D images of fruit on trees is presented. Several sequences of RGB-D images were taken in an orchard by a hand held PrimeSense Carmine 1.09 short range sensor operating in $100 \mu \mathrm{m}$-mode. The image resolution was $320 \times 240$. From these sequences four sets of images were selected, each set containing images of one of the following fruit sorts: plum, nectarine, pear and tomato. One of the criteria for image selection was that the images had been taken while the camera was steady or moving very slowly in order to avoid motion blur or misalignment of depth and RGB image. The other criterion was that the fruit in the image is not deep in a shadow so that it can be unambiguously distinguished by visual inspection. Although the evaluated method uses only depth images, RGB images are used for visual inspection of the results by a human evaluator and therefore, clear visibility of the fruit in the image is very important in order for the evaluator to be able to correctly categorize the results obtained by the evaluated approach.

The investigated segmentation approach described in Section III was applied to the four sets of acquired RGB-D

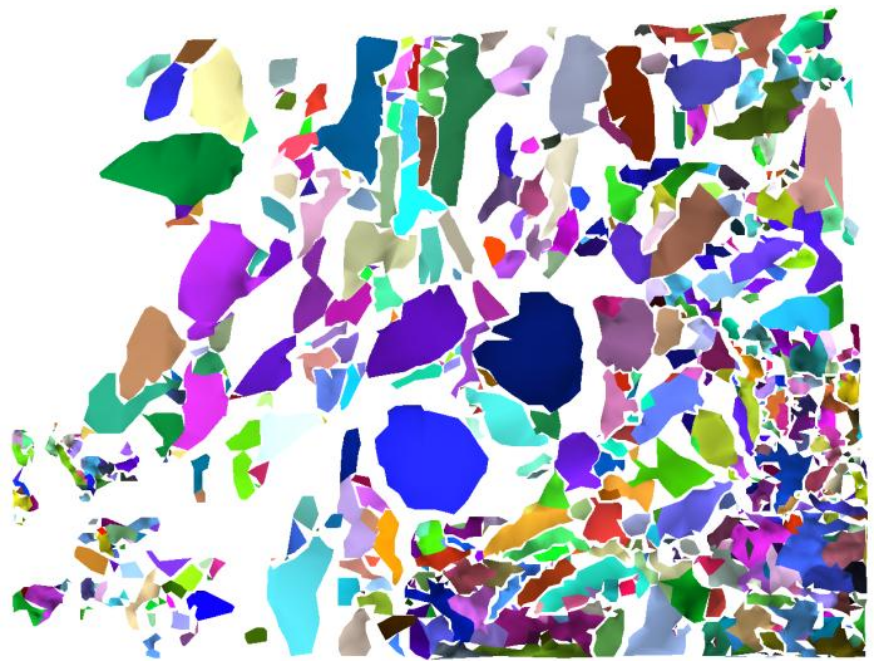

Fig. 2. Segmentation of the triangular mesh shown in Fig. 1. (c) into approximately convex surfaces. Each segment is displayed in a different color. 
images. Since the precision of the applied sensor degrades with the distance, the tested algorithm was configured to consider only the points of the acquired point clouds within the distance of $0.6 \mathrm{~m}$ from the camera. Hence, in the following, the term point cloud refers to this close subset of an original point cloud. The threshold for generating triangular meshes from the depth images was set to $2 \mathrm{~mm}$, which means that the maximum distance between any point in the point cloud and the closest triangle of the mesh in the direction of the camera optical axis is $2 \mathrm{~mm}$ at most. The segmentation threshold $\varepsilon$ explained in Section III was set to $5 \mathrm{~mm}$.

The obtained segmentation results were visually inspected by human evaluators which categorized all segments representing fruits into three categories:

$\mathrm{C} 1$ : the fruit is represented by a single segment where the boundary of this segment is very close to the fruit boundary;

C2: the fruit is represented by two or more segments, where the convex hull of these segments is very close to the fruit boundary;

C3: the fruit is represented by a segment whose boundary extends over a significant portions of adjacent objects.

The results of this analysis are presented in TABLE I.

Examples of three considered segmentation categories of possible segmentation result are shown in Fig. 3. For illustrative purposes, one segment is manually selected between all segments obtained by the evaluated approach in each example. In the top row a correct segmentation is shown. It can be seen in the 3D display (right) that although two pears are positioned very close to one another, the tested segmentation algorithm separated them into two segment according to their approximately convex shape. The middle row of Fig. 3. is an example of oversegmentation, where the visible surface of the tomato is represented by two segments because of a concavity appearing on the top of the fruit. An example of false segmentation, where a fruit is merged together with adjacent leafs into one segment, is shown in the bottom row of Fig. 3. In this example, the plum is positioned among leafs in such a way that its surface aligns with their surfaces forming an approximately convex surface.

The average execution time on a standard PC with an Intel Core i5-3230M CPU at $2.6 \mathrm{GHz}$ measured over a sequence of 1433 images was $0.114 \mathrm{~s}$.

From the presented analysis it can be concluded that there are a high percentage of samples where fruits are merged with adjacent leaves or branches (C3). Such merging occurs e.g. in the case where a leaf covers a fruit tightly so that the union of the point sets representing the fruit and the leaf is an approximately convex surface. This indicates that additional cues such as color and shape constraints must be used in order to achieve reliable segmentation. Furthermore, a significant percentage of fruit samples are oversegmented (C2). This can be caused by small concavities in the fruit shape or sensor noise. These effects could be reduced by increasing the segmentation threshold $\varepsilon$. This would, however, increase the percentage of undersegmentation (C3). The applied
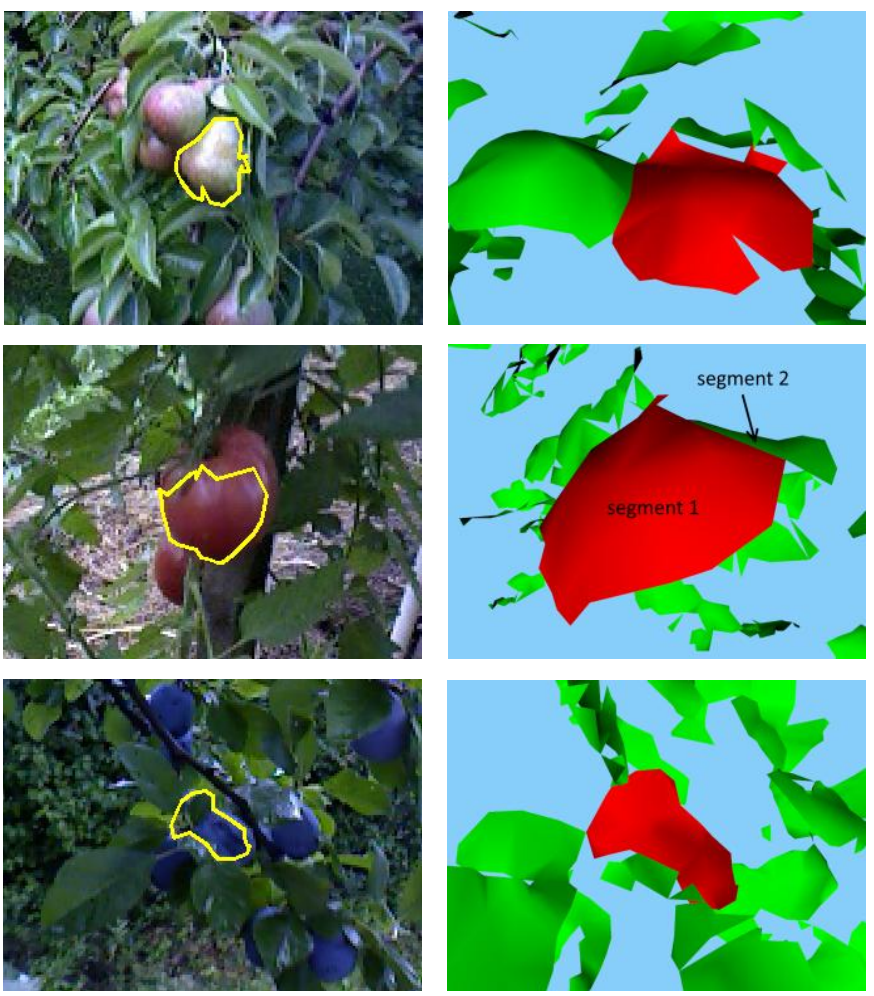

Fig. 3. Sample results of segmentation to approximately convex surfaces. Segments representing fruits are outlined by yellow contours in RGB images (left) and displayed by red color (right). Examples of a correct segmentation (top), oversegmentation (middle) and merging of fruit with adjacent objects (bottom) are presented. Notice that the tomato in the middle row is represented by two segments denoted by segment 1 and segment 2 .

segmentation method is very sensitive to the choice of the parameter $\varepsilon$ and, therefore, its value should be adjusted by an optimization procedure, which is not considered in this paper.

\section{CONCLUSION}

The purpose of the research reported in this paper was to determine whether the segmentation of point clouds acquired by a 3D camera into approximately convex surfaces has a potential to be used as a preprocessing step of a fruit recognition system as well as to identify its drawbacks. The obtained experimental results demonstrate that a relatively high percentage of fruit in a close range of $0.6 \mathrm{~m}$ from the camera can be detected by the studied method as separate segments. However, oversegmentation as well as merging with adjacent leaves or branches also appears very frequently. In order to cope with the oversegmentation problem a higher-level

TABLE I. SEGMENTATION RESULTS

\begin{tabular}{|c|c|c|c|c|c|}
\cline { 4 - 6 } \multicolumn{2}{c}{} & \multicolumn{3}{c|}{ Result Category } \\
\hline Fruit sort & \#images & \#fruits & $\boldsymbol{C 1}(\boldsymbol{\%})$ & $\boldsymbol{C 2}(\boldsymbol{\%})$ & $\boldsymbol{C 3}(\boldsymbol{\%})$ \\
\hline plum & 99 & 653 & 76.6 & 4.0 & 19.5 \\
\hline nectarine & 50 & 147 & 66.7 & 11.6 & 21.8 \\
\hline pear & 61 & 407 & 71.7 & 7.9 & 20.4 \\
\hline tomato & 38 & 96 & 59.4 & 9.4 & 31.3 \\
\hline
\end{tabular}


algorithm which would group adjacent segments according to their color and shape features can be applied. Furthermore, the undersegmentation could be reduced by introducing a color cue in the segmentation framework. Parameterized shape models of particular fruit sorts can be used for grouping of adjacent segments as well as for splitting the segments resulting from false merging of fruits with adjacent objects.

The investigation reported in this paper can be considered as a preliminary research in the development of a fruit recognition system which uses an RGB-D camera as the sensor and performs segmentation of the acquired point cloud into primitives before applying a suitable classifier. A more accurate evaluation of this approach can be made by testing it in combination with state-of-the-art classifiers.

\section{REFERENCES}

[1] R. Cupec, E. K. Nyarko, D. Filko, "Fast 2.5D Mesh Segmentation to Approximately Convex Surfaces, " Proc. of the 5th European Conference on Mobile Robots (ECMR), pp. 127-132, Örebro, Sweden, 2011

[2] R. C. Harrell, D. C. Slaughter and P. D. Adsit, "A Fruit-Tracking System for Robotic Harvesting, " Machine Vision and Applications, vol. 2, issue 2, pp 69-80, 1989.

[3] T. Kataok, Y. Ot and T. Hirom, "A Segmentation Algorithm for the Automatic Recognition of Fuji Apples at Harvest, " Biosystems engineering, vol. 83, issue 4, December 2002.

[4] J. Lu, N. Sang, Y. Hua and H. Fu, "Detecting citrus fruits with highlight on tree based on fusion of multi-map, “ Optik - International Journal for Light and Electron Optics, vol. 125, issue 8, pp. 1903-1907, April 2014.

[5] H. Li, W. S. Lee and K. Wang, "Identifying blueberry fruit of different growth stages using natural outdoor color images, "Computers and Electronics in Agriculture vol. 125, pp. 91-101, August 2014.

[6] H. Peng, X. Zou, J. Xiong, Y. Chen, A. Guo and K. Chen, "Recognition of Mature Citrus in Natural Scene under the Occlusion Condition, " Journal of Information \& Computational Science, vol. 11, issue 6, pp. 1947-1958, April 2014.

[7] L. Qiang, C. Jianrong, L. Bin, D. Lie and Z. Yajing, "Identification of fruit and branch in natural scenes for citrus harvesting robot using machine vision and support vector machine, "International Journal of Agricultural and Biological Engineering, vol. 7, no. 2, pp. 115-121, 2014.

[8] E. A. Murillo-Bracamontes, M. E. Martinez-Rosas, M. M. MirandaVelasco, H. L. Martinez-Reyes, J. R. Martinez-Sandoval and H. Cervantes-de-Avila, "Implementation of Hough transform for fruit image segmentation, "Procedia Engineering, vol. 35, pp. 230-239, 2012.

[9] R. Roscher, K. Herzog, A. Kunkel, A. Kicherer, R. Töpfer and W. Förstner, "Automated image analysis framework for high-throughput determination of grapevine berry sizes using conditional random fields, " Computers and Electronics in Agriculture, vol. 100, pp. 148158, January 2014.

[10] S. Sengupta and W. S. Lee, "Identification and determination of the number of immature green citrus fruit in a canopy under different ambient light conditions, "Biosystems Engineering, vol. 117, pp. 51-61, January 2014.

[11] Y. Song, C. A. Glasbey, G. W. Horgan, G. Polder, J. A. Dieleman, G. and W. A. M. van der Heijden, "Automatic fruit recognition and counting from multiple images, " Biosystems Engineering, vol. 118, pp. 203-210, February 2014

[12] S. Cubero, M. P. Diago, J. Blasco, J. Tardáguila, B. Millán and N. Aleixosd "A new method for pedicel-peduncle detection and size assessment of grapevine berries and other fruits by image analysis, " Biosystems Engineering, vol. 117, pp. 62-72, January 2014.

[13] W. Jia, D. Zhao, F. Cheng, B. Xua, Y. Zhanga and J. Wang, "Automatic recognition vision system guided for apple harvesting robot, "
Computers \& Electrical Engineering, vol. 38, issue 5, pp. 1186-1195, September 2012.

[14] E. Kelman and R. Linker, "Vision-based localisation of mature apples in tree images using convexity, " Biosystems Engineering, vol. 118, pp. 174-185, February 2014.

[15] D. Stajnko, M. Lakota and M. Hočevar, "Estimation of number and diameter of apple fruits in an orchard during the growing season by thermal imaging, "Computers and Electronics in Agriculture, vol. 42, issue 1, pp. 31-42, January 2004.

[16] D. M. Bulanon, T. F. Burks and V. Alchanatis, "Image fusion of visible and thermal images for fruit detection, " Biosystems Engineering, vol. 113, pp. 12-22, May 2009.

[17] D. Font, T. Pallejà, M. Tresanchez, D. Runcan, J. Moreno, D. Martínez, M. Teixidó and J. Palacín, "A Proposal for Automatic Fruit Harvesting by Combining a Low Cost Stereovision Camera and a Robotic Arm, “ Sensors, vol. 14, issue 7, pp. 11557-11579, 2014.

[18] J. Rakun, D. Stajnko and D. Zazula, "Detecting fruits in natural scenes by using spatial-frequency based texture analysis and multiview geometry, "Computers and Electronics in Agriculture, vol. 76, issue 1, pp. 80-88, March 2011.

[19] R. Xiang, H. Jiang and Y. Ying, "Recognition of clustered tomatoes based on binocular stereo vision, "Computers and Electronics in Agriculture, vol. 106, pp. 75-90, August 2014.

[20] A. Plebe and G. Grasso, "Localization of spherical fruits for robotic harvesting, "Machine Vision and Applications, vol. 13, issue 2, pp. 7079, November 2001

[21] A. R. Jiménez, R. Ceres and J. L. Pons, "A vision system based on a laser range-finder applied to robotic fruit harvesting, " Machine Vision and Applications, vol. 11, issue 6, pp. 321-329, May 2000.

[22] K. Tanigaki, T. Fujiura, A. Akase and J. Imagawa, "Cherry-harvesting robot, "Computers and Electronics in Agriculture, vol. 63, issue 1, pp. 65-72, August 2008.

[23] A. R. Jiménez, R. Ceres and J. L. Pons, "A Survey of Computer Vision Methods for Locating Fruit on Trees, "Transaction of the ASAE, vol. 43 issue 6, pp. 1911-1920, 2000.

[24] A. R. Jiméneza, A. K. Jain, R. Ceres and J. L. Pons, "Automatic fruit recognition a survey and new results using Range - Attenuation images, "Pattern Recognition, vol. 32, issue 10, pp. 1719-1736, October 1999.

[25] K. Kapach, E. Barnea, R. Mairon, Y. Edan and O. Ben-Shahar, "Computer vision for fruit harvesting robots - state of the art and challenges ahead, "International Journal of Computational Vision and Robotics vol. 3, issue 1/2, pp. 4-34, April 2012.

[26] P. Li, S. Lee and H. Hsu, "Review on fruit harvesting me thod for potential use of automatic fruit harvesting systems, " Procedia Engineering vol. 23, pp. 351-366, 2011.

[27] F. Schmitt and X. Chen, "Fast segmentation of range images into planar regions, " In IEEE Computer Society Conf. on Computer Vision and Pattern Recognition (CVPR), pp. 710-711, 1991.

[28] A. Aldoma, F. Tombari, L. Di Stefano, and M. Vincze, "A global hypothesis verification method for $3 \mathrm{~d}$ object recognition," Proc. of the European Conference on Computer Vision (ECCV), pp. 511-524, Florince, Italy, 2012.

[29] J. Tang, S. Miller, A. Singh, and P. Abbeel, "A textured object recognition pipeline for color and depth image data," Proc. of the International Conference on Robotics and Automation (ICRA), pp. 3467-3474, Saint Paul, US, 2012.

[30] C. Papazov and D. Burschka, "An efficient ransac for 3D object recognition in noisy and occluded scenes," Proc. of the 10th Asian Conference on Computer Vision (ACCV), pp. 135-148, Queenstown, New Zealand, 2010

[31] M. Attene, M. Mortara, M. Spagnuolo and B. Falcidieno, "Hierarchical Convex Approximation of 3D Shapes for Fast Region Selection", Computer Graphics Forum, vol. 27, issue 5, pp. 1323-1332, 2008. 